\title{
UTILIZAÇÃO DA MACRÓFITA EGERIA DENSA NA BIOSORÇÃO DO CORANTE REATIVO 5G
}

\author{
Aparecido Nivaldo Módenes* \\ Fernando Rodolfo Espinoza* \\ Vanessa Lizeria Alflen* \\ Andréia Colombo* \\ Carlos Eduardo Borba*
}

\begin{abstract}
Resumo: Neste trabalho foi avaliado o potencial de biossorção da biomassa seca da macrófita aquática Egeria densa na remoção do corante reativo azul $5 \mathrm{G}$ presente em efluentes de indústrias têxteis. O teste cinético e de equilíbrio foram realizados em sistema batelada sob agitação constante, com pH e temperatura controlados. Os resultados obtidos na cinética de biossorção demonstram uma rápida remoçáo do corante reativo azul 5G, com tempo de equilíbrio em torno de 120 minutos e taxa de remoçáo de aproximadamente $95 \%$. O modelo cinético de pseudo-segunda ordem foi o que melhor representou os dados experimentais. No estudo do equilíbrio, verificou-se que o modelo de Langmuir foi o que melhor se ajustou aos dados experimentais, apresentando capacidade máxima de biossorção de $29,12 \mathrm{mg} \mathrm{g}^{-1}$ e razão entre as taxas de sorção e dessorção de $0,13 \mathrm{~L} \mathrm{~g}^{-1}$. Os resultados obtidos demonstram que a macrófita $E$. densa tem potencial para ser utilizada em sistemas de tratamento de efluentes industriais.
\end{abstract}

Palavras-chaves: Biossorção, corante têxtil, Egeria densa

\begin{abstract}
In this work, Egeria densa biomass has been used as an alternative adsorbent for removal of reactive blue $5 \mathrm{G}$ dye from an aqueous solution. Batch kinetic and equilibrium adsorption experiments were carried out using a rotary shaker, under controlled sorption temperature, and constant dye solution $\mathrm{pH}$. Biosorption kinetic results have shown a quickly dye removal in the first minutes, achieving the equilibrium time at 120 min with a $95 \%$ dye removal. Kinetic adsorption data were better fitted by the pseudo-second order kinetic model. The equilibrium adsorption data were better represented by the Langmuir isotherm model, showing a maximum adsorption capacity of $29.12 \mathrm{mg} \mathrm{g}^{-1}$ and an adsorption affinity of $0.13 \mathrm{Lg}^{-1}$. Based on these results, the $E$. densa is expected to be used as a good biosorbent in treatment systems of dying effluents.
\end{abstract}

Key-words: biossorption, textile dye, Egeria densa

\section{INTRODUÇÁO}

O crescimento das atividades industriais tem provocado inúmeros problemas ambientais devido à poluição e degradação de ecossistemas, gerando acúmulo de poluentes tais como: metais pesados, compostos sintéticos, resíduos nucleares, etc. Dentre os setores industriais, a indústria têxtil é considerada uma das mais poluidoras, devido ao elevado volume e variação de composiçáo de seus efluentes (Vandevivere et al., 1998).
Alguns dos principais componentes dos efluentes têxteis são os corantes, que são compostos químicos orgânicos que possuem a propriedade de absorver luz visível seletivamente, razão pela qual aparecem coloridos, devido à presença de grupos cromóforos tais como nitro, nitroso, azo e carbonila. A cor destes compostos é intensificada e/ou modificada por grupos auxocromos tais como etila, nitro, amino, sulfônico, hidroxila, metóxi, etó$\mathrm{xi}$, cloro e bromo. Os corantes reativos constituem uma classe de corantes utilizados em escala crescente

\footnotetext{
* Centro de Engenharias e Ciências exatas, Programa de Pós-Graduação em Engenharia Química da Universidade Estadual do Oeste do Paraná
} 
pelas indústrias têxteis devido à sua reatividade com as fibras e estabilidade da cor (Kimura et al., 1999).

A remoçáo da cor dos efluentes têxteis é um dos grandes problemas ambientais apresentado por esse setor. A elevada estabilidade biológica destes produtos dificulta seu tratamento por via convencional (Dallago et al., 2005).

Diversos métodos convencionais têm sido utilizados no tratamento de efluentes contaminados com corantes, dentre eles citam-se a coagulação, precipitação, filtração, ozonição, processos oxidativos avançados e eletrofloculaçáo (Guaratini e Zanoni, 2000, Mohan et al., 2002, Kunz et al., 2002, Palácio et al., 2009, Manentti et al., 2010). O processo da biossorção que emprega como adsorvente materiais de origem natural surge como uma tecnologia promissora e em atual expansão. Este método se torna atraente devido ao seu baixo custo, fácil disponibilidade, eficiência elevada, biodegradabilidade, facilidade de operação e capacidade para tratar corantes em formas mais concentradas (Meshko et al., 2001; Sanghi $e$ Bhattacharya 2002; Bulut e Aydin, 2006).

Dentre os diversos materiais utilizados como biomassa, as macrófitas têm se destacado pela boa capacidade de remoçáo de poluentes e pela abundância, pois é considerada a principal planta daninha aquática em diversas partes do mundo (Bini e Thomaz, 2005; Módenes et al., 2009a; Módenes et al., 2009b). Estas plantas são consideradas infestantes e nocivas, e a produção de biossorventes utilizando estas plantas pode ser realizada com somente custos de secagem e transporte.

O aproveitamento da biomassa de plantas aquáticas como adsorventes naturais para o controle da poluiçáo ambiental tem sido muito pouco explorado, principalmente no Brasil. Desta forma, este trabalho tem por objetivo avaliar o potencial da biomassa seca da macrófita Egeria densa na remoção de corante reativo azul 5G.

\section{MATERIAIS E MÉTODOS}

\subsection{MATERIAIS}

A biomassa da macrófita Egeria densa utilizada como biossorvente foi coletada no lago de Itaipu no estado do Paraná. Em seguida foi lavada com água corrente, enxaguada com água destilada, seca à temperatura ambiente e triturada em moinho (Tecnal), sem separaçáo por granulometria, para a utilizaçáo nos experimentos.
Foi utilizado neste trabalho, soluções de corante com concentraçóes na faixa de 25 a $80 \mathrm{mg}$ $\mathrm{L}^{-1}$, preparadas a partir do corante reativo azul $5 \mathrm{G}$ (Texpal Química), cuja representação da molécula é apresentada na Fig. 1.

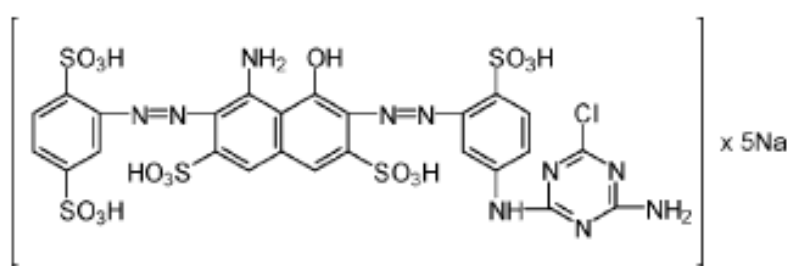

Figura 1. Estrutura química do corante reativo azul 5G (Koprivanac et al., 2005).

\subsection{PROCEDIMENTO EXPERIMENTAL}

Para aprimorar as condiçóes experimentais de adsorção, realizou-se um teste preliminar de investigação do efeito do $\mathrm{pH}$ na solução. Para este teste, foi adicionado $250 \mathrm{mg}$ da macrófita Egeria densa a $50 \mathrm{~mL}$ de solução de corante reativo azul $5 \mathrm{G}\left(80 \mathrm{mg} \mathrm{L}^{-1}\right)$, com os valores de $\mathrm{pH}$ ajustados de 1 a 10 , em duplicata. O teste foi realizado sob agitação constante em "shaker" com temperatura controlada de $30^{\circ} \mathrm{C}$ por um período de $120 \mathrm{~min}$. As concentraçóes das amostras foram determinadas em espectrofotômetro UV-VIS.

O estudo da cinética de biossorção foi realizado utilizando-se erlenmeyers de $125 \mathrm{~mL}$, nos quais adicionou-se $250 \mathrm{mg}$ da biomassa seca da macrófita Egeria densa e $50 \mathrm{~mL}$ da solução de corante reativo azul $5 \mathrm{G}$ com concentração inicial de $25 \mathrm{mg} \mathrm{L}^{-1}$, previamente preparada. O teste cinético foi realizado em duplicata, com temperatura controlada de $30^{\circ} \mathrm{C}$ e ajuste de $\mathrm{pH} 1$, sob agitação. As amostras foram coletadas em intervalos de tempo pré-definidos e em seguida filtradas. As concentraçōes das amostras foram analisadas em espectrofotômetro UV-VIS.

$\mathrm{Na}$ determinação do equilíbrio de sorção fez-se o uso de erlenmeyers de $125 \mathrm{~mL}$, com valores de biomassa da macrófita variando de 10 a $50 \mathrm{mg}$ e adição de volumes de solução de corante reativo azul $5 \mathrm{G}$ de $50 \mathrm{~mL}$ com concentrações iniciais variando de 35 a $70 \mathrm{mg} \mathrm{L}^{-1}$. Os experimentos foram conduzidos sob agitação constante por um período de 120 min., mantendo-se a temperatura controlada de $30^{\circ} \mathrm{C}$ e $\mathrm{pH} 1$. Após o período de tempo determinado as amostras foram retiradas, filtradas e analisadas em espectrofotômetro UV-VIS. A quan- 
tidade de corante adsorvido pela macrófita foi determinada pelo balanço de massa dado pela Eq. 1 .

$$
q=\frac{V\left(C_{0}-C\right)}{m}
$$

Em que: $q q$ é a quantidade de corante adsorvida pela biomassa da macrófita $\left(\mathrm{mg} \mathrm{g}^{-1}\right) ; C_{0}$ $C_{0}$ e $C C$ são as concentraçóes iniciais e finais do corante nas soluçôes, respectivamente $\left(\mathrm{mg} \mathrm{L}^{-1}\right) ; V$ $V$ é o volume da soluçáo de corante $(50 \mathrm{~mL}) ; \mathrm{mm}$ é a massa de adsorvente (g).

\section{RESULTADOS E DISCUSSÓES}

\subsection{EFEITO DO PH}

Os resultados do teste preliminar realizado e o ajuste pela função do tipo logística, mostraram que o $\mathrm{pH}$ é um importante parâmetro no fenômeno de adsorção. A influência do $\mathrm{pH}$ na adsorção do corante azul reativo 5G é mostrada na Fig. 2.

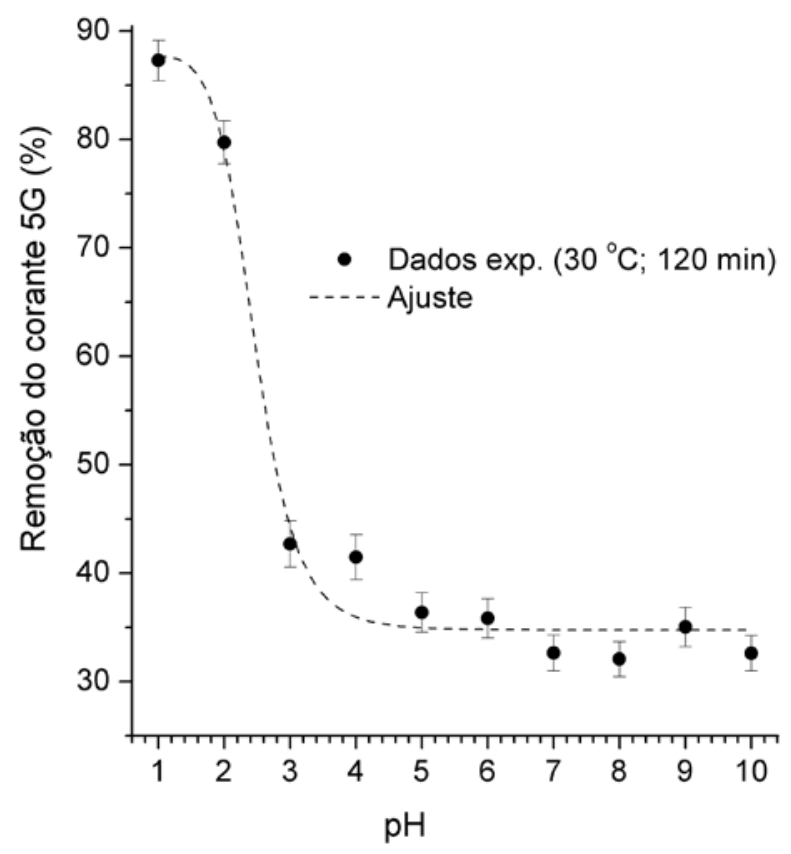

Figura 2. Efeito do $\mathrm{pH}$ na adsorção de corante azul reativo $5 \mathrm{G}$.

O potencial de remoção de corante reativo azul 5G pela macrófita Egeria densa aumenta com o decréscimo do $\mathrm{pH}$, apresentando remoção aproximada de 87\%, utilizando-se $\mathrm{pH} 1$.

De acordo com Fiorentin et al. (2010), quando o $\mathrm{pH}$ da soluçáo de corante diminui, mais prótons estão disponíveis na soluçáo de corante e o número de sítios ativos carregados negativamente é reduzido, assim, os prótons são conduzidos para a superfície do adsorvente, que é composta princi- palmente por grupos funcionais tais como amino e carboxila, para ser mais positivamente carregada. $\mathrm{O} \mathrm{pH}$ também pode afetar a estabilidade estrutural do corante reativo azul 5G, pois, sob uma condição ácida, a molécula do corante pode ser desprotonada no seio da solução, resultando numa molécula polar $\left(\mathrm{R}-\mathrm{SO}_{3}\right)$, com uma alta densidade de carga negativa. Portanto, a repulsão eletrostática entre o sítio adsorvente e os íons de corante carregados negativamente foi reduzida em $\mathrm{pH}$ baixo. Consequentemente, os grupos funcionais carregados positivamente poderiam exercer uma forte atração eletrostática em moléculas aniônicas de corante. Outro modo de adsorção também pode estar presente (troca iônica ou quelação, por exemplo). Além disso, uma abundância de sítios superficiais carregados negativamente é esperada para valores de $\mathrm{pH}$ neutro e alcalino, causando um efeito desfavorável sobre uma adsorção aniônica de corante, porque uma repulsáo eletrostática na superfície do adsorvente está atuando e, assim, a eficiência de adsorção do corante cai para 30-42\%.

\subsection{PARÂMETROS CINÉTICOS}

Os resultados experimentais do teste cinético, bem como, o ajuste por uma função do tipo logística são apresentados na Fig. 3.

Os dados cinéticos demonstraram que houve uma grande redução da concentração de corante logo nos primeiros minutos e atingiu-se a concentração de equilíbrio em torno de 120 minutos, com remoção de aproximadamente 95\%.

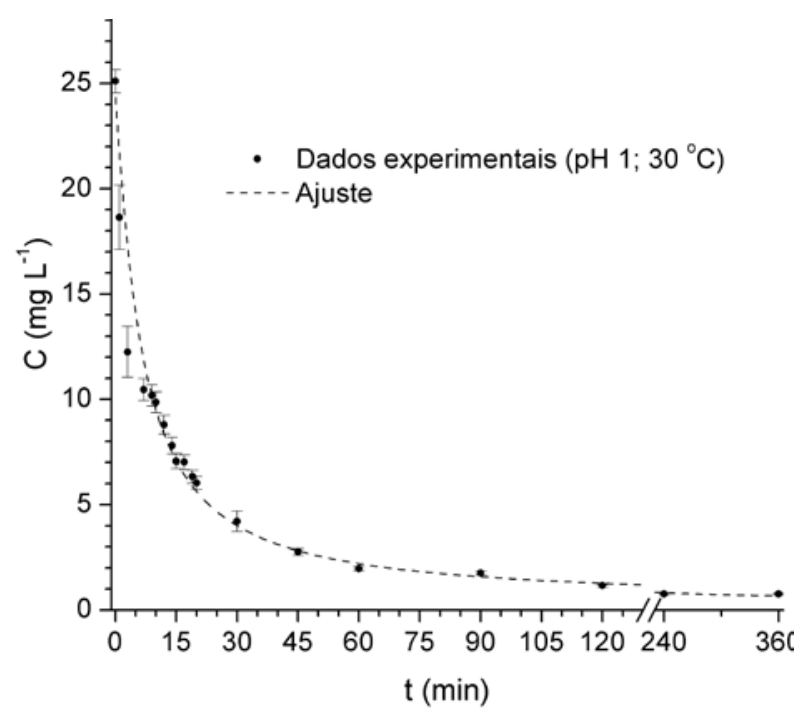

Figura 3. Cinética de biossorção do corante reativo azul $5 \mathrm{G}$, para os dados experimentais obtidos em $\mathrm{pH} 1$ e $30^{\circ} \mathrm{C}$. 
$\mathrm{Na}$ avaliação dos dados cinéticos utilizaram-se as equaçóes de Lagergren e Ho. A equação de Lagergren é conhecida a mais tempo, descrevendo a taxa de adsorção em função da capacidade de adsorção. A expressáo de Ho inclui a quimiossorção e foi aplicada com sucesso na adsorção de metais, íons, corantes, herbicidas, óleos e substâncias orgânicas a partir de soluçôes aquosas (Ho, 2006).

A forma linear do modelo de pseudo-primeira ordem descrito por Lagergren é apresentado pela Eq. 2:

$$
\log \left(q_{q_{1}}-q_{t}\right)=\log \left(q_{q}\right)-\left(\frac{K_{1}}{2,303}\right) t
$$

Sendo, $K_{1} K_{1}$ a constante de velocidade $\left(\mathrm{min}^{-1}\right)$; $q_{e q} q_{e q}$ a quantidade de corante sorvido no equilíbrio ( $\left.\mathrm{mg} \mathrm{g}^{-1}\right) ; q_{t} q_{t}$ a quantidade de corante sorvido no tempo $t t\left(\mathrm{mg} \mathrm{g}^{-1}\right)$.

Para o modelo linear de pseudo-segunda ordem, segundo Ho (2006) tem-se a Eq. 3:

$$
\frac{t}{q_{t}}=\frac{1}{K_{2} q_{q}^{2}}+\frac{1}{q_{q}} t
$$

Em que: $K_{2} K_{2}$ é a constante de velocidade $\left(\mathrm{g} \mathrm{mg}^{-1} \mathrm{~min}^{-1}\right)$.

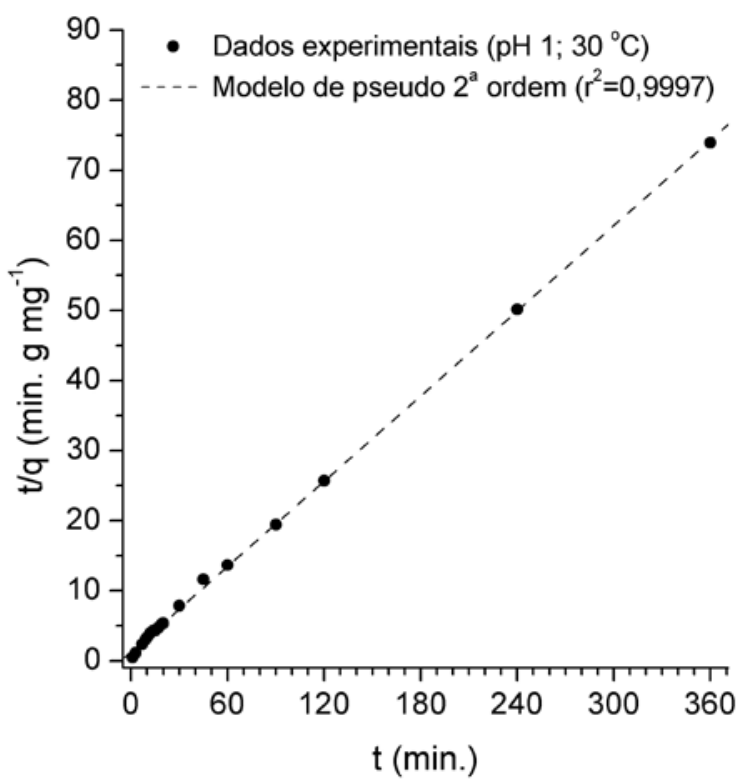

Figura 4. Modelo de pseudo-segunda ordem do processo de biossorçáo do corante reativo azul $5 \mathrm{G}$ para os dados experimentais obtidos em $\mathrm{pH} 1$ e $30^{\circ} \mathrm{C}$.

O modelo de pseudo-segunda ordem de Ho, mostrado na Figura 4, foi o que melhor se ajustou aos dados experimentais, com coeficien- te de ajuste $0,9997, q_{\text {eq }}$ igual a $4,932 \mathrm{mg} \mathrm{g}^{-1}$ e $\mathrm{K}_{2}$ igual a $0,031 \mathrm{~g} \mathrm{mg}^{-1} \mathrm{~min}^{-1}$. $\mathrm{O}$ modelo de pseudo-primeira ordem apresentou coeficiente de ajuste $-0,9307$, demonstrando ser inadequado aos dados experimentais.

\subsection{PARÂMETROS DE EQUILÍBRIO DE ADSORÇÃO}

Os dados de equilíbrio de sorção foram avaliados por isotermas de adsorção. Estas isotermas representam uma relação de equilíbrio entre a concentração de corante na fase líquida e a quantidade de corante com uma massa biosorvente para uma temperatura específica. A isoterma é muito importante para estimar a máxima capacidade de adsorçáo e afinidade entre adsorbato e adsorvente, além de outros parâmetros físicos (Fiorentin et al., 2010). Uma vez que a resposta do fenômeno de adsorção está relacionada ao tipo de material utilizado como adsorvente e as características físicas e químicas possuídas pelo adsorvato, bem como as condiçóes experimentais de adsorção, um número de processos de adsorção de poluentes têm sido estudados na tentativa de encontrar uma explicaçáo adequada para os mecanismos e cinética para classificar as soluções ambientais (Weber, 1963).

As isotermas aplicadas são descritas segundo os modelos apresentados por Langmuir, Freundlich e Temkin.

O modelo de Langmuir é representado pela Eq. 4:

$$
q_{q}=q_{\max } \frac{b_{q}}{1+b_{q}}
$$

Em que: $q_{\theta q} q_{\theta q}$ é a quantidade de corante adsorvido por unidade de massa de biomassa em equilíbrio $\left(\mathrm{mg} \mathrm{g}^{-1}\right) ; c_{\theta q} c_{\theta q}$ é a concentração de corante em solução não adsorvido em equilíbrio $\left.(\mathrm{mg} \mathrm{L})^{-1}\right) ; q_{\max } q_{\max }$ é a capacidade máxima de sorção do corante por unidade de massa de biomassa para formar uma monocamada completa na superfície $\left(\mathrm{mg} \mathrm{g}^{-1}\right) ; b b$ é a razão entre a constante cinética de adsorção e a constante cinética de dessorção, $\left(\mathrm{L} \mathrm{mg}^{-1}\right)$.

Os dois parâmetros $b$ e $q_{\max }$ são constantes da isoterma de Langmuir, sendo que o parâmetro $b$ representa a razão entre as taxas de sorção e dessorção. $\mathrm{O}$ parâmetro $q_{\max }$ representa o número total de sítios disponíveis no material biossorvente, 
ou seja, indica a completa saturação da superfície do sorvente (cobertura da monocamada).

Para representação do modelo de Freundlich tem-se a Eq. 5:

$$
q_{q}=k\left(C_{q}\right)^{n}
$$

Em que: $k$ é uma constante relacionada à capacidade de adsorção; $n$ é uma constante relacionada à intensidade de adsorção; $C_{e q}$ é a concentração de corante em solução não adsorvido em equilíbrio, $\left(\mathrm{mg} \mathrm{L}^{-1}\right) ; q_{e q}$ é a quantidade de corante adsorvido por unidade de massa de biomassa em equilíbrio $\left(\mathrm{mg} \mathrm{g}^{-1}\right)$.

A isoterma de Freundlich é uma equação empírica que considera a existência de uma estrutura em multicamadas, e não prevê a saturação da superfície baseada no processo de adsorção. Assim, o modelo permite a existência de uma cobertura superficial infinita. Valores de $k$ maiores indicam que o adsorvente utilizado apresenta maior potencial para a captaçáo do adsorvato encontrado em solução.

O modelo Temkin considera os efeitos das interaçóes indiretas entre as moléculas do adsorvato e a diminuição dos calores de adsorçáo com o aumento da taxa de remoção. Assumindo que o calor de adsorção das moléculas na camada diminui linearmente com o preenchimento da superfície do adsorvente. A isoterma de Temkin é descrita pela Eq. 6 .

$$
q_{e_{1}}=B \cdot \mathrm{h}\left(k_{T} C_{q}\right)
$$

Em que: B é a constante de Temkin que relaciona o calor de adsorção com o número total de sítios $\left(\mathrm{mg} \mathrm{g}^{-1}\right)$ e $\mathrm{k}_{\mathrm{T}}$ é a constante de Temkin $\left(\mathrm{L} \mathrm{mg}^{-1}\right)$.

As isotermas de Langmuir, Freundlich e Temkin, representadas pelas Equaçóes 4-6, com dois parâmetros, foram ajustadas aos dados experimentais de equilíbrio para o corante reativo $5 \mathrm{G}$. Os parâmetros de cada isoterma foram estimados utilizando o software Origin ${ }^{\circledR}$, versáo 8.0. As isotermas juntamente com os dados experimentais de equilíbrio para o corante reativo $5 \mathrm{G}$, em $\mathrm{pH} 1,30$ ${ }^{\circ} \mathrm{C}$ e 120 minutos, são apresentados na Figura 5.

$\mathrm{Na}$ Tabela 1 são mostrados os dados da análise estatística o os valores dos parâmetros para os modelos. De acordo com o coeficiente de correlação, verifica-se que o modelo de isoterma de Langmuir foi o que se ajustou melhor aos dados de equilíbrio seguido pelo modelo de Temkin e Freundlich.

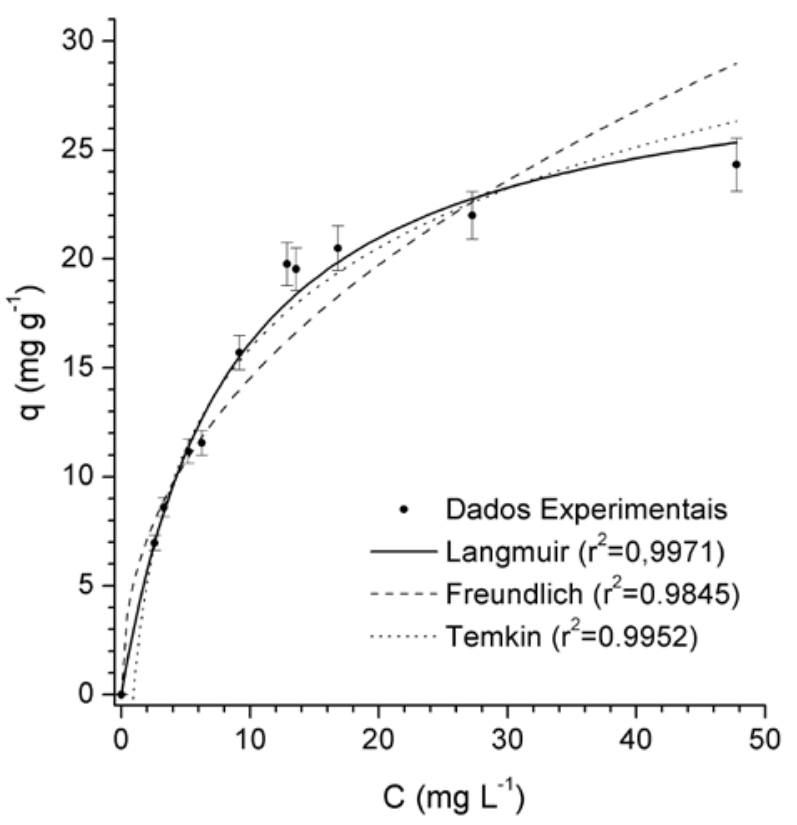

Figura 5 Ajuste das isotermas de Langmuir, Freundlich e Temkin e dos dados experimentais da adsorçáo de corante reativo azul $5 \mathrm{G}$ pela macrófita $E$. densa para os dados experimentais obtidos em $\mathrm{pH} 1,30^{\circ} \mathrm{C}$ e $120 \mathrm{~min}$.

Tabela 1 Ajuste dos parâmetros das isotermas de Langmuir, Freundlich e Temkin para os dados experimentais obtidos em $\mathrm{pH} 1,30^{\circ} \mathrm{C}$ e 120 min.

\begin{tabular}{|c|c|c|}
\hline Modelos & Parâmetros & valores \\
\hline Langmuir & $q_{\max }\left(\mathrm{mg} \mathrm{g}^{-1}\right)$ & $29,83 \pm 1,32$ \\
& $b\left(\mathrm{~L} \mathrm{mg}^{-1}\right)$ & $0,12 \pm 0,01$ \\
& $\mathrm{r}^{2}$ & 0,9971 \\
& $\chi^{2}$ & 1,1656 \\
\hline Freundlich & $k\left(\mathrm{mg} \mathrm{g}^{-1}\right)$ & $5,67 \pm 0,53$ \\
& $n$ & $0,44 \pm 0,04$ \\
& $\mathrm{r}^{2}$ & 0,9845 \\
& $\chi^{2}$ & 6,1726 \\
\hline Temkin & $k_{T}\left(\mathrm{~L} \mathrm{mg}^{-1}\right)$ & $1,09 \pm 0,11$ \\
& $B\left(\mathrm{mg} \mathrm{g}^{-1}\right)$ & $6,66 \pm 0,36$ \\
& $\mathrm{r}^{2}$ & 0,9952 \\
& $\chi^{2}$ & 1,8990 \\
\hline
\end{tabular}

Para o sistema estudado, a alta capacidade de adsorção $\left(\mathrm{q}_{\max }=29,83 \pm 1,32 \mathrm{mg} \mathrm{g}^{-1}\right)$, baixo tempo de equilíbrio (120 min.) e baixo custo de preparação, sugerem que a macrófita aquática $E$. densa pode ser utilizada como um biossorvente al- 
ternativo na remoção de corantes em sistema de tratamento de efluentes têxteis.

\section{CONCLUSÃO}

Através desta pesquisa verificou-se que a macrófita Egeria densa possui grande potencial de remoção do corante reativo azul $5 \mathrm{G}$ presente em efluentes têxteis industriais, visto que houve uma redução significativa na quantidade de corante presente na solução, em torno de $60 \%$, após os primeiros minutos e aproximadamente $95 \%$ no equilíbrio. $\mathrm{O}$ modelo cinético de pseudo-segunda ordem foi o que obteve ajuste satisfatório aos dados experimentais.

O controle de $\mathrm{pH}$ é importante no processo de adsorção do corante reativo $5 \mathrm{G}$, pois em $\mathrm{pH}$ ácido existe uma alta atração eletrostática entre a superfície do adsorvente carregada positivamente e o corante aniônico.

As isotermas de Langmuir, Freundlich e Temkin apresentaram comportamento favorável na interpretação dos dados experimentais de equilíbrio, com valores de $q_{\max }$ igual a $29,83 \mathrm{mg} \mathrm{g}^{-1}$ para Langmuir, $k_{T}$ igual a $1,09 \mathrm{~L} \mathrm{mg}^{-1}$ para Temkin, e $k$ igual a 5,67 $\mathrm{mg} \mathrm{g}^{-1}$ para Freundlich. Baseado nos valores do coeficiente de correlação, a isoterma de Langmuir foi a que melhor representou os dados experimentais de equilíbrio.

\section{REFERÊNCIAS}

Bulut Y., Aydın H., 2006. A kinetics and thermodynamics study of methylene blue adsorption on wheat shells. Desalination. 194, pp. 259-267.

Bini, L.M.; Thomaz, S.M., 2005. Prediction of Egeria najas and Egeria densa occurrence in a large subtropical reservoir (Itaipu Reservoir, Brazil Paraguay). Aquatic botany. 83 n. 3, pp. 227-238.

Dallago R. M., Smaniotto A., Oliveira L. C. A., 2005. Resíduos sólidos de curtumes como adsorventes para a remoção de corantes em meio aquoso. Quimica Nova. 28, n.3, pp. 433-437.

Fiorentin, L.D., Trigueros, D.E.G., Módenes, A.N., Espinoza-Quiñones, F.R., Pereira, N.C., Barros, S.T.D., Santos, O.A.A., 2010. Biosorption of reactive blue $5 \mathrm{G}$ dye onto drying orange bagasse in batch system: Kinetic and equilibrium modeling. Chemical Engineering Journal. 163, pp. 68-77.

Guaratini, C. C. I., Zanoni, M. V. B., 2000. Revisão: Corantes Têxteis. Química Nova. 23, n.1, pp. 71-78

Ho, Y. S., 2006. Review of second-order models for adsorption systems. Journal of Hazardous $M a$ terials. 136 pp. 681-689.

Kimura, I. Y., Gonçalves Jr., A. C., Stolberg, J., Laranjeira, M. C. M., Fávere, V. T., 1999. Efeito do $\mathrm{pH}$ e do tempo de contato na adsorção de corantes reativos por microesferas de quitosana. Polímeros: Ciência e Tecnologia. pp. 51-57.

Koprivanac, N. Kusic, H., Vujevi, D., et al., 2005. Influence of iron on degradation of organic dyes in corona. Journal of Hazardous Materials $117 \mathrm{pp}$. 113-119.

Kunz A., Zamora P. P. Moraes, S. G., Durán N., 2002. Revisão: Novas Tendências no Tratamento de Efluentes Têxteis. Química Nova. 25, n.1, pp. 78-82.

Manenti, D. R., Gomes, L.F.S., Borba, F.H., Módenes, A.N., Espinoza-Quiñones, F.R., Palácio, S.M., 2010. Otimização do processo foto-Fenton utilizando irradiaçáo artificial na degradação do efluente têxtil sintético. Engevista (UFF) v. 12, n1, pp 22 a 32.

Meshko V., Markovska L., Mincheva M., Rodrigues A.E., 2001. Adsorption of basic dyes on granular activated carbon and natural zeolite. $\mathrm{Wa}$ ter Research. 35, pp. 3357-3366.

Módenes, A.N., Pietrobelli, J.M.T.A., EspinozaQuiñones, F.R., 2009a. Cadmium biosorption by non-living aquatic macrophytes Egeria densa. Water Science and Technology. 60 pp. 293-300.

Módenes, A.N., Pietrobelli, J.M.T.A., Espinoza-Quiñones, F.R., Suzaki, P.Y.R., Alflen, V.L., Fagundes-Klen, M.R., 2009b. Potencial de biossorção do zinco pela macrófita Egeria densa. Engenharia Sanitária e Ambiental. 14, pp. 465-470. 
Mohan D., Singh K.P., Singh G., Kumar K., 2002. Removal of Dyes from Wastewater Using Flyash, a Low-Cost adsorvent. Industrial \& Engineering Chemical Research. 41 pp. 3688-3695.

Palácio, S.M., Espinoza-Quiñones, F.R., Módenes, A.N., Oliveira, C.C., Borba, F.H. Silva Jr., F.G, 2009. Toxicity assessment from electro-coagulation treated-textile dye wastewaters by bioassays. Journal of Hazardous Materials. 172 pp. 330-337.

Sanghi R., Bhattacharya B., 2002. Review on decolorisation of aqueous dye solutions by low cost adsorbents. Coloration Technology. 118, pp 256269.

Vandevivere, P. C., Bianchi, R., Verstraete, W., 1998, Treatment and Reuse of Wastewater from the textile wetprocessing industry: Review of emerging technologies, Journal of Chemical Technology \&. Biotechnology 72, pp. 289-302.

Weber W.J., Morriss J.C., 1963. Kinetics of adsorption on carbon from solution, Journal Sanitary Engineering Division, American Society of Civil Engineers. 89 pp. 31-60. 